\title{
Many-polaron description of impurities in a Bose-Einstein condensate in the weak-coupling regime
}

\author{
W. Casteels, ${ }^{1}$ J. Tempere, ${ }^{1,2}$ and J. T. Devreese ${ }^{1}$ \\ ${ }^{1}$ TQC, Universiteit Antwerpen, Groenenborgerlaan 171, B-2020 Antwerpen, Belgium \\ ${ }^{2}$ Lyman Laboratory of Physics, Harvard University, Cambridge, Massachusetts 02138, USA
}

(Received 5 September 2011; published 9 December 2011)

\begin{abstract}
The weak-coupling many-polaron formalism is applied to the case of the polaronic system consisting of impurities in a Bose-Einstein condensate. This allows investigating the ground-state properties and the response of the system to Bragg spectroscopy. Then, this theory is applied to the system of spin-polarized fermionic lithium-6 impurities in a sodium condensate. The Bragg spectrum reveals a peak that corresponds to the emission of Bogoliubov excitations. Both the ground-state properties and the response spectrum show that the polaronic effect vanishes at high densities. We also look at two possibilities to define the polaronic effective mass and observe that this results in a different quantitative behavior if multiple impurities are involved.
\end{abstract}

DOI: 10.1103/PhysRevA.84.063612

PACS number(s): $67.85 . \mathrm{Pq}$

\section{INTRODUCTION}

Quantum gases have revealed themselves as excellent quantum simulators for many-body theories from condensedmatter physics and, in particular, to experimentally examine strong-coupling regimes that are not attainable with solidstate experiments [1]. Recently, it was shown that, when the Bogoliubov approximation is valid, the system of impurities in a Bose-Einstein condensate (BEC) can be added to this list through a mapping onto the Fröhlich polaron Hamiltonian [2,3]. The Fröhlich solid-state polaron consists of a charge carrier (electron, hole) interacting with the longitudinal optical phonons in an ionic crystal or a polar semiconductor [4,5]. The Fröhlich polaron Hamiltonian has resisted an exact analytical diagonalization and has been submitted to many approximation methods (a review on the Fröhlich solid-state polaron can be found in Ref. [6]). The polaronic effect of an impurity in a BEC has been the subject of several recent theoretical papers. Some examples are the polaronic effects in optical lattices [7-9], the application of the Feynman variational path-integral technique to study the ground-state properties [10], an extension of this technique to examine the response properties [11], and a strong-coupling approximation [12]. This revealed that the static properties are characterized by the ratio of the masses of the bosons and the impurity together with the polaronic-coupling parameter $\alpha$, defined as

$$
\alpha=\frac{a_{I B}^{2}}{\xi a_{B B}},
$$

with $a_{I B}$ as the impurity-boson scattering length, $a_{B B}$ as the boson-boson scattering length, and $\xi$ as the healing length of the condensate. As a function of this coupling parameter, two polaronic regimes were identified, reminiscent of the acoustic polaron [13]. In the context of the Fröhlich solid-state polaron, the intermediate- and strong-coupling regimes still supply important theoretical challenges, but the weak-coupling regime is well understood. The weak- and intermediatecoupling theories of Lee et al. [14] were extended by Lemmens et al. [15] to the case of an interacting polaron gas. Similarly, the optical absorption theory of Devreese et al. [16] was extended to the case of interacting polarons in Ref. [17] by two of the present authors. In these extensions, the effect of the interactions between the polarons is taken into account through the structure factor of the electron gas. The goal of this paper is to apply a similar generalization to extend the polaronic theory of a single impurity in a Bose condensate to the case of a dilute gas of interacting impurities. This is needed since an experimental realization of the BEC-impurity polaron will involve multiple impurities and will require the use of Feshbach resonances to reach the strong-coupling regime [10].

Within the Bogoliubov approximation, the Hamiltonian of $N$ impurities in a condensate can be mapped onto the sum of the mean-field energy $E_{M F}$ and the Fröhlich $N$-polaron Hamiltonian $\widehat{H}_{\text {pol }}^{N}[10]$. The mean-field energy is given by

$$
E_{M F}=E_{G P}+N_{0} N U_{I B}(\vec{q}=\overrightarrow{0})
$$

where the first term is the Gross-Pitaevskii energy of the condensate [18] and the second term is the interaction shift due to the impurities with $N_{0}$ as the number of condensed bosons and $U_{I B}(\vec{q})$ as the Fourier transform of the impurity-boson interaction potential. The polaron Hamiltonian describes the mutual interaction between the impurities and their interaction with the Bogoliubov excitations,

$$
\begin{aligned}
\widehat{H}_{\mathrm{pol}}^{N}= & \sum_{i}^{N} \frac{\widehat{\vec{p}}_{i}^{2}}{2 m_{I}}+\sum_{\vec{k}} \hbar \omega_{\vec{k}} \widehat{a}_{\vec{k}}^{\dagger} \widehat{a}_{\vec{k}}+\sum_{\vec{k}} \sum_{i}^{N}\left(V_{\vec{k}} \widehat{a}_{\vec{k}} e^{i \vec{k} \cdot \widehat{\vec{r}}_{i}}\right. \\
& \left.+V_{\vec{k}}^{\dagger} \widehat{a}_{\vec{k}}^{\dagger} e^{-i \vec{k} \cdot \widehat{\vec{r}}_{i}}\right)+\frac{1}{2} \sum_{i \neq j} v\left(\widehat{\vec{r}}_{i}-\widehat{\vec{r}}_{j}\right) .
\end{aligned}
$$

The first term represents the kinetic energy of the impurities with mass $m_{I}$ and position (momentum) operators $\widehat{\vec{r}}_{i}\left(\widehat{\vec{p}}_{i}\right)$, the second term gives the kinetic energy of the Bogoliubov excitations with dispersion $\omega_{\vec{k}}$ and creation (annihilation) operators $\widehat{a}_{\vec{k}}^{\dagger}\left(\widehat{a}_{\vec{k}}\right)$, the third term is the interaction between the impurities and the Bogoliubov excitations with interaction amplitude $V_{\vec{k}}$, and the fourth term represents the mutual interaction between the impurities with $v(\vec{r})$ as the interaction potential. The Bogoliubov dispersion is given by

$$
\omega_{\vec{k}}=c k \sqrt{1+(\xi k)^{2} / 2},
$$


where the speed of sound in the condensate was introduced $c=$ $\hbar /\left(\sqrt{2} m_{B} \xi\right)$ with $m_{B}$ as the mass of the bosons. The interaction amplitude is given by

$$
V_{\vec{k}}=\sqrt{N_{0}}\left[\frac{(\xi k)^{2}}{(\xi k)^{2}+2}\right]^{1 / 4} g_{I B},
$$

where a contact potential was assumed for the impurity-boson interaction potential $U_{I B}(\vec{q})=g_{I B}$. At low temperatures, the amplitude $g_{I B}$ is completely determined by the reduced mass $m_{r}=\left(m_{B}^{-1}+m_{I}^{-1}\right)^{-1}$ and the impurity-boson scattering length $a_{I B}, g_{I B}=2 \pi \hbar^{2} a_{I B} / m_{r}$.

Another important consideration is the stability of the mixture against phase separation. At zero temperature, in Refs. [19] and [20], it was shown that the mixture is stable if the following inequality is satisfied:

$$
n^{1 / 3} \leqslant \frac{(6 \pi)^{2 / 3}}{12 \pi} \frac{m_{r} a_{B B}}{m_{B} m_{I} a_{I B}^{2}},
$$

with $n$ as the density of the impurities.

Since the impurities are not charged, the response cannot be studied through optical absorption measurements as for the Fröhlich solid-state polaron. In Ref. [11], it was shown that the internal excitation structure of the BEC-impurity polaron can be probed with Bragg spectroscopy, which is a technique that has proven to be very successful in the study of BECs (see, for example, Refs. [21] and [22]). The experimental setup consists of two laser beams with wave vectors $\vec{k}_{1}$ and $\vec{k}_{2}$ and energies $\omega_{1}$ and $\omega_{2}$, which are impinged on the impurities. Then, these can absorb a photon from one beam and can emit it in the other beam, which results in the exchange of a wave vector $\vec{k}=\vec{k}_{1}-\vec{k}_{2}$ and an energy $\hbar \omega=\hbar \omega_{1}-\hbar \omega_{2}$ to the impurities. The response of the system, after an exposure during a time interval $\tau$, can be measured by counting the number of atoms $N_{\text {Bragg }}$ that have gained a wave vector $\vec{k}$ as a function of $\omega$, which, in the formalism of linear-response theory, is given by [18]

$$
N_{\text {Bragg }}=\frac{2}{\hbar}\left(\frac{V}{2}\right)^{2} \tau \operatorname{Im} \chi(\vec{k}, \omega)
$$

with $V$ as the amplitude of the laser-induced potential and $\chi(\vec{k}, \omega)$ as the density-response function, defined as

$$
\chi(\vec{k}, \omega)=\frac{i}{\hbar} \int d t e^{i \omega t}\left\langle\rho_{\vec{k}}(t) \rho_{\vec{k}}^{\dagger}\right\rangle
$$

In the following, we start by summarizing the main results of Refs. [15] and [17] on the weak-coupling treatment of the Fröhlich solid-state $N$-polaron system together with an indication of what changes if one considers impurities in a BEC. We also show two different ways to define the polaronic effective mass. Then, this formalism is applied for spin-polarized fermionic impurities, and the results are examined as a function of the impurity density and the exchanged momentum for the Bragg response for lithium- 6 impurities in a sodium condensate.

\section{WEAK-COUPLING TREATMENT OF THE MANY-POLARON GAS}

\section{A. Ground-state properties of the many-polaron gas and the inertial effective mass}

The weak-coupling variational method as introduced in Ref. [14] by Lee et al. for the description of a single polaron was generalized in Ref. [15] to the case of a many-polaron system. With this purpose, the following variational wave function was introduced:

$$
\left|\Psi_{L D B}\right\rangle=\widehat{U}|0\rangle|\psi\rangle
$$

with $|0\rangle$ as the vacuum wave function for the bosonic excitations, $|\psi\rangle$ as the wave function of the impurities, and $\widehat{U}$ as a canonical transformation of the form

$$
\widehat{U}=\exp \left\{\sum_{i}^{N} \sum_{\vec{k}}\left[f_{\vec{k}} \widehat{a}_{\vec{k}} e^{i \vec{k} \cdot \widehat{\vec{r}}_{i}}-f_{\vec{k}}^{*} \widehat{a}_{\vec{k}}^{\dagger} e^{-i \vec{k} \cdot \widehat{\vec{r}}_{i}}\right]\right\},
$$

where $\left\{f_{\vec{k}}\right\}$ are variational functions. Minimizing the expectation value of the $N$-polaron Hamiltonian (3) with respect to the variational wave function (9) as a function of $\left\{f_{\vec{k}}\right\}$ results in the following expression for the ground-state energy:

$$
\begin{aligned}
\frac{E}{N}= & \varepsilon_{\text {kin }}+\frac{1}{2} \sum_{\vec{k}} v(\vec{k})[S(\vec{k})-1] \\
& -\sum_{\vec{k}} \frac{S^{2}(\vec{k})\left|V_{\vec{k}}\right|^{2}}{\hbar \omega_{\vec{k}} S(\vec{k})+\frac{\hbar^{2} k^{2}}{2 m_{I}}},
\end{aligned}
$$

where $\varepsilon_{\text {kin }}$ is the kinetic energy per particle, $\varepsilon_{\text {kin }}=$ $N^{-1}\left\langle\psi\left|\sum_{i}^{N} \frac{\hat{p}_{i}^{2}}{2 m_{I}}\right| \psi\right\rangle$, and $S(\vec{k})$ is the static structure factor of the impurities,

$$
S(\vec{k})=1+\frac{1}{N}\left\langle\psi\left|\sum_{i \neq j}^{N} e^{i \vec{k} \cdot\left(\vec{r}_{i}-\vec{r}_{j}\right)}\right| \psi\right\rangle .
$$

Another important property of the polaronic system is the inertial effective mass of the polarons $m^{(\mathrm{in})}$. Within the one-polaron weak-coupling formalism, $m^{\text {(in) }}$ was calculated in Ref. [14]. The generalization for many polarons allows a determination of this effective mass, which is related to the many-polaron system as a whole and is called the inertial effective mass in the following. The total momentum $\overrightarrow{\mathcal{P}}=\sum_{i} \vec{p}_{i}+\sum_{\vec{k}} \hbar \vec{k} \widehat{a}_{\vec{k}}^{\dagger} \widehat{a}_{\vec{k}}$ commutes with the Hamiltonian (3) and, thus, is a conserved quantity. This conserved quantity can be introduced explicitly in the minimization process by means of a Lagrange multiplier $\vec{v}$, which corresponds to the velocity of the polaronic system,

$$
\widehat{H}_{\mathrm{pol}}^{N}(\vec{v})=\widehat{H}_{\mathrm{pol}}^{N}-\vec{v} \cdot\left(\sum_{i} \widehat{\vec{p}}_{i}+\sum_{\vec{k}} \hbar \vec{k} \widehat{a}_{\vec{k}}^{\dagger} \widehat{a}_{\vec{k}}-\overrightarrow{\mathcal{P}}\right) .
$$

Minimization of the expectation value of Eq. (13) with respect to Eq. (9) as a function of $\left\{f_{\vec{k}}\right\}$ and $\vec{v}$ together with an Taylor expansion for small $\vec{v}$, results in

$$
\overrightarrow{\mathcal{P}}=N m_{I} \vec{v}+\frac{2}{3} \hbar^{2} N \sum_{\vec{k}} \frac{\left|V_{\vec{k}}\right|^{2} S^{2}(k)}{\left[\hbar \omega_{\vec{k}} S(k)+\frac{\hbar^{2} k^{2}}{2 m_{I}}\right]^{3}} k^{2} \vec{v},
$$


where isotropy of the system was assumed. We can now identify the inertial effective mass $m^{\text {(in) }}$ through the expression $\overrightarrow{\mathcal{P}}=N m^{(\text {in) }} \vec{v}$, which relates the speed of the polaron to its momentum, i.e.,

$$
m^{\text {(in) }}=m_{I}+\frac{2}{3} \hbar^{2} \sum_{\vec{k}} \frac{\left|V_{\vec{k}}\right|^{2} S^{2}(k)}{\left[\hbar \omega_{\vec{k}} S(k)+\frac{\hbar^{2} k^{2}}{2 m_{I}}\right]^{3}} k^{2} .
$$

Note that the inertial effective mass (15) is a property of the whole polaron system. This is important for an experiment where the behavior of all the impurities together is studied. For example, when one studies the collective oscillation of the impurities in a harmonic trap, the resulting effective oscillation frequency is a function of this inertial effective mass. This effect has been used to experimentally determine the effective mass of Fermi polarons in Ref. [23].

Note that, for the ground-state properties, the presence of multiple impurities is completely described through the static structure factor of the impurities. In the limit of vanishing density, the static structure factor becomes 1 , and the expressions for the energy and the effective mass of a single polaron from Ref. [14] are retrieved.

\section{B. Bragg response of the many-polaron gas and the spectral effective mass}

In Ref. [17], the optical absorption of the $N$-polaron gas consisting of electrons interacting with phonons was calculated within the weak-coupling formalism. The polaronic system consisting of impurities in a BEC cannot be probed with optical absorption, but instead, an appropriate experimental technique is Bragg spectroscopy as shown in Ref. [11]. This means that a finite-momentum exchange has to be taken into account, which is negligible in the case of the optical absorption calculation. Furthermore, it is the density-density correlation function (8) that determines the response instead of the current-current correlation, which is needed for the optical absorption. However, these two are related closely since the application of two partial integrations transforms Eq. (8) into a correlation function of the time derivative of the density $\dot{\rho}_{\vec{k}}$ after which one can use the Fourier transform of the continuity equation $\left(\dot{\rho}_{\vec{k}}=i \vec{k} \cdot \vec{j}_{\vec{k}}\right)$ to obtain a current-current correlation function,

$$
\chi(\omega, \vec{k})=-\frac{i}{\hbar \omega^{2}} \int_{0}^{\infty} d t e^{i \omega t}\left\langle\left[\vec{k} \cdot j_{\vec{k}}(t), \vec{k} \cdot j_{\vec{k}}^{\dagger}\right]\right\rangle .
$$

For the calculation of Eq. (16), we use the derivation of Ref. [17], which is based on the wave function (9) and which preserves only terms of the lowest order in the coupling amplitude $\left|V_{\vec{k}}\right|^{2}$. The main difference of the present result is the incorporation of a finite-momentum exchange $\vec{k}$. This results in the following expression for the imaginary part of the density-response function (16), which is proportional to the Bragg response (7),

$\operatorname{Im} \chi(\omega, \vec{k})=\frac{1}{\omega^{4}} \frac{\pi}{m_{I}^{2}} \sum_{\vec{q}}(\vec{k} \cdot \vec{q})^{2}\left|V_{\vec{q}}\right|^{2} S\left(\vec{q}+\vec{k}, \omega-\omega_{\vec{q}}\right)$,

where $S(\vec{k}, \omega)$ is the dynamic structure factor of the impurities,

$$
S(\vec{k}, \omega)=\frac{1}{2 \pi \hbar} \int_{-\infty}^{\infty} d t e^{i \omega t}\left\langle\psi\left|\rho_{\vec{k}}(t) \rho_{\vec{k}}^{\dagger}\right| \psi\right\rangle .
$$

The response also allows a determination of an effective mass, which we call the spectral effective mass and denote by $m^{\text {(spec) }}$. This is performed through an extension of the $f$-sum rule that was introduced in Ref. [24] and was generalized for the polaron in a condensate in Ref. [11] as

$$
\frac{N \pi}{2 m^{\text {(spec })}}+\int_{0^{+}}^{\infty} d \omega \omega \lim _{\vec{k} \rightarrow 0} \frac{\operatorname{Im}[\chi(\vec{k}, \omega)]}{k^{2}}=\frac{N \pi}{2 m_{I}} .
$$

The origin of this sum rule is a $\delta$ peak at $\omega=0$ in the response, and the spectral weight of this $\delta$ peak in the $\vec{k} \rightarrow 0$ limit equals the first term in Eq. (19). It is important to note that the spectral effective mass as determined by Eq. (19) is a single-particle

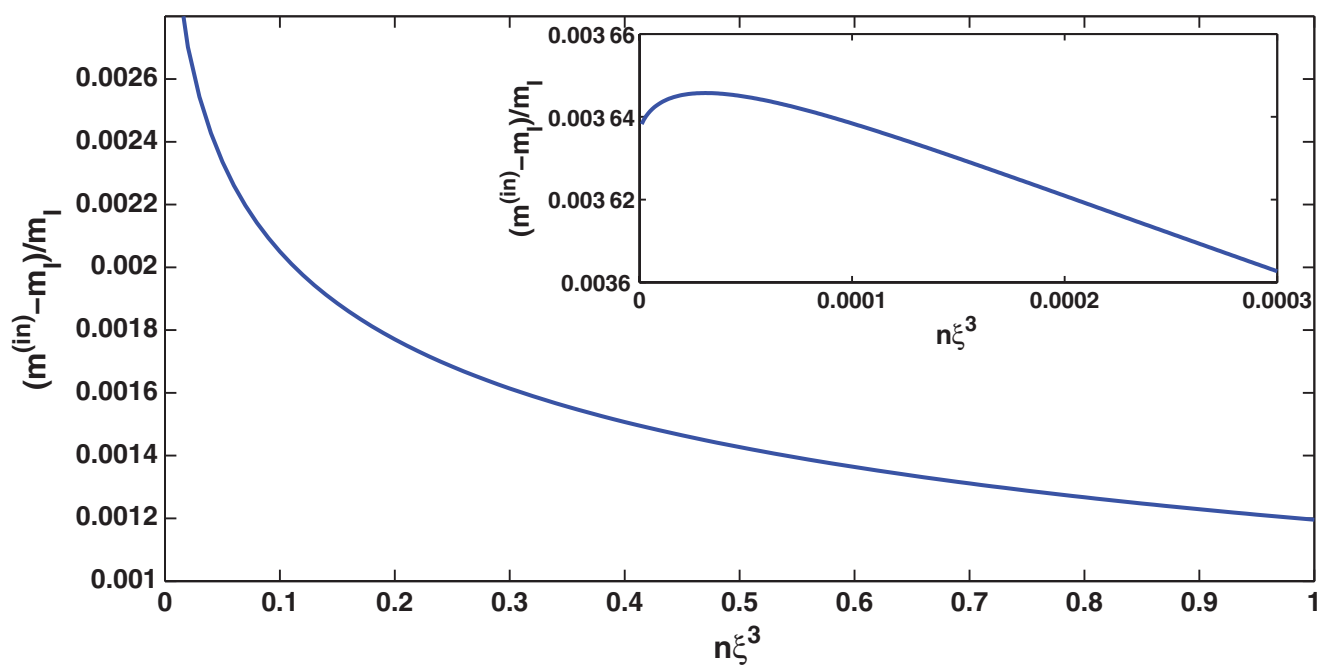

FIG. 1. (Color online) The inertial effective mass of the polarons as a function of the density for lithium- 6 impurities in a sodium condensate at $\alpha=0.01$ according to formula (22). The inset shows the behavior at small densities. In the limit $n \rightarrow 0$, the effective mass of a single polaron is retrieved, and for $n \rightarrow \infty$, the effective mass becomes the bare-impurity mass. 


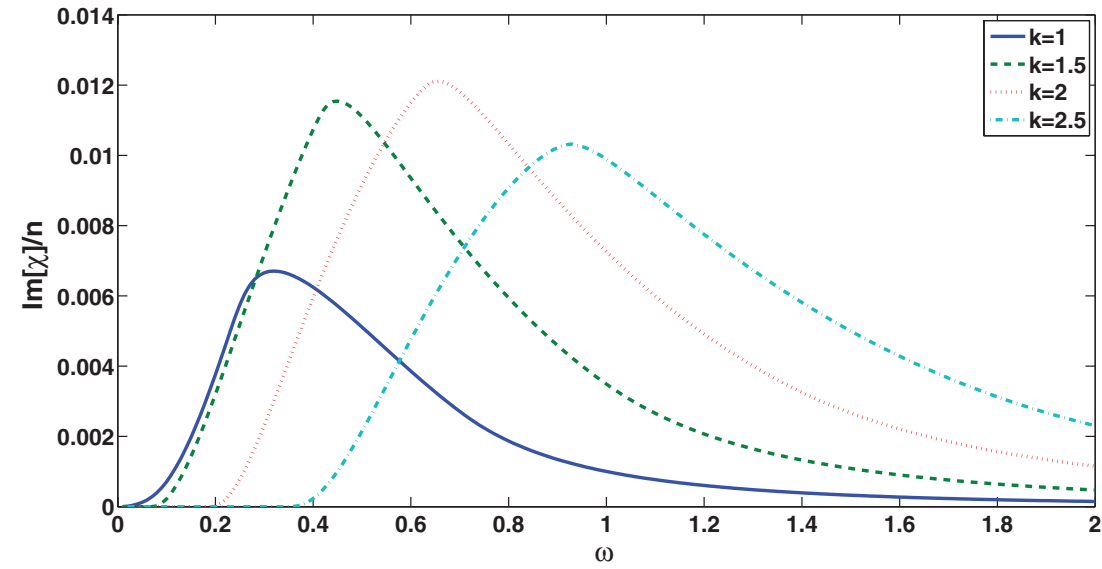

FIG. 2. (Color online) The Bragg response (24) of the polaronic system consisting of polarized lithium- 6 impurities in a sodium condensate as a function of $\omega$ for different values of the exchanged momentum $k$. The impurity density is taken as $n=0.01$, and the polaronic-coupling parameter is $\alpha=0.01$. Everything is in polaronic units, i.e., $\hbar=m_{I}=\xi=1$. property and, thus, is not the same as the inertial effective mass of expression (15). Only in the limit of one polaron, do the two masses coincide as can be checked from Eq. (19). This polaronic spectral effective mass, as defined in Eq. (19), was, for example, measured in Ref. [25] for the Fröhlich solid-state polaron.

Note that, in this formalism, the influence of multiple impurities on the polaronic response is determined solely by the dynamic structure factor of the impurities.

\section{SPIN-POLARIZED FERMIONIC IMPURITY GAS IN A BEC}

In this section, we apply the results of the previous section to the case of a spin-polarized gas of fermionic impurities. Because of the Pauli exclusion principle, the $s$-wave scattering length is zero, which results in essentially no interactions between the impurities at low temperatures. This means the impurities can be described as an ideal fermionic gas for which the static and dynamic structure factors are known. All results in this section are presented in polaronic units, i.e., $\hbar=m_{I}=\xi=1$, and for lithium- 6 impurities in a sodium condensate, i.e., $m_{B} / m_{I} \approx 3.82207$.

\section{A. Ground-state properties}

The static structure factor of an ideal fermionic gas at zero temperature is given by [26]

$$
S(\vec{k})= \begin{cases}\frac{3}{2} \frac{k}{2 k_{F}}-\frac{1}{2}\left(\frac{k}{2 k_{F}}\right)^{3}, & \text { if } k<2 k_{F}, \\ 1, & \text { if } k \geqslant 2 k_{F},\end{cases}
$$

where $k_{F}$ is the Fermi wave vector, which, for a nondegenerate gas, is given by $k_{F}=\left(6 \pi^{2} n\right)^{1 / 3}$ with $n$ as the impurity density. Introducing the kinetic energy $\varepsilon_{\text {kin }}$ of an ideal fermionic gas together with the Bogoliubov dispersion (4) and the interaction amplitude (5) in the expression for the ground-state energy (11) leads to

$$
\begin{aligned}
\frac{E}{N}= & \frac{3}{10} k_{F}^{2}-\frac{\alpha}{2 \pi}\left(\frac{m_{B}+1}{m_{B}}\right)^{2} \int_{0}^{\infty} d k k^{2} \\
& \times\left[\frac{m_{B} S^{2}(\vec{k})}{k \sqrt{k^{2}+2} S(\vec{k})+m_{B} k^{2}}\left(\frac{k^{2}}{k^{2}+2}\right)^{1 / 2}-\frac{m_{B}}{m_{B}+1}\right],
\end{aligned}
$$

with $\alpha$ as the polaronic-coupling parameter from expression (1). The first term represents the kinetic energy, and the second is the polaronic contribution; the interaction energy has vanished since we are describing an ideal gas. Notice that, in the polaronic contribution, an additional term appeared, which was needed to obtain a convergent energy and was obtained in Ref. [10] as a result of the renormalization of the boson-impurity interaction. The polaronic contribution in Eq. (21) grows linearly with $k_{F}$ as the number of particles is increased. Since the kinetic energy of the Fermi gas grows as $k_{F}^{2}$, the relative contribution of the polaronic energy with respect to the kinetic energy decreases. In the limit of high densities, the kinetic energy dominates the polaronic effect.

Introducing the Bogoliubov dispersion (4) and the interaction amplitude (5) in the expression for the polaron inertial effective mass (15) results in

$$
\begin{aligned}
m^{(\text {in })}= & 1+\frac{4 \alpha}{3 \pi}\left(\frac{m_{B}+1}{m_{B}}\right)^{2} \int_{0}^{\infty} d k \frac{1}{\sqrt{2+k^{2}}} \\
& \times \frac{k^{2} S^{2}(\vec{k})}{\left[\frac{1}{m_{B}} \sqrt{k^{2}+2} S(\vec{k})+k\right]^{3}} .
\end{aligned}
$$

This expression is presented in Fig. 1 as a function of the impurity density. In the limit $n \rightarrow 0$, the one-polaron result is retrieved, which already was anticipated using formula (15). For $n \rightarrow \infty$, the inertial effective mass becomes equal to the bare-impurity mass. This limit can easily be examined analytically with Eq. (22), which reveals a $n^{-1 / 3}$ behavior at high densities. For the Fröhlich solid-state polaron, the same qualitative behavior as in Fig. 1 was found for the effective mass if the electrons are described as a free gas $[27,28]$.

\section{B. Response to Bragg spectroscopy}

For the response, the dynamic structure factor is needed, which, for an ideal fermionic gas at temperature zero, is given 

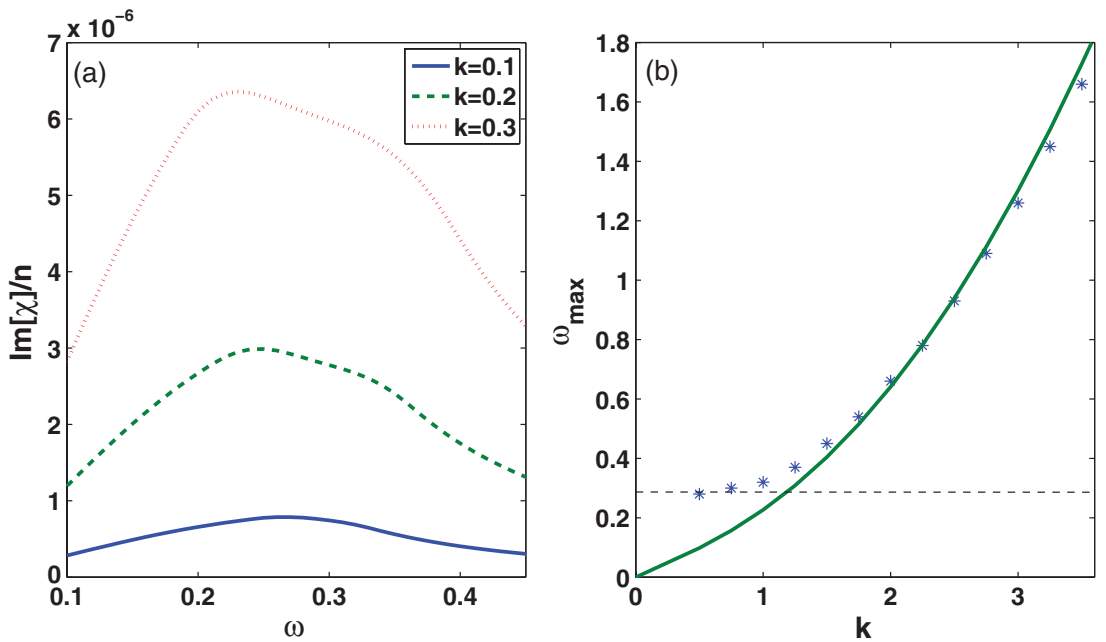

FIG. 3. (Color online) In (a), the Bragg response (24) is shown for the polaronic system consisting of polarized lithium- 6 impurities in a sodium condensate as a function of $\omega$ for different values of the exchanged momentum $k$ at small $k$, showing the shoulder structure on the peak. In (b), the position of the maximum of the peak in the Bragg response is presented (markers) together with the Bogoliubov spectrum (4) (full line) and the Fermi frequency $\omega_{F}$ (dotted line). In both plots, the impurity density is $n=0.01$, and the polaronic-coupling parameter is $\alpha=0.01$. Everything is in polaronic units, i.e., $\hbar=m_{I}=\xi=1$.

by [29]

$$
S(\vec{q}, \omega)=\frac{1}{4 \pi^{2} q} \theta(\omega)\left\{\begin{array}{lll}
\omega, & \text { if } \quad \frac{k_{F}^{2}}{2}>\frac{1}{2 q^{2}}\left(\omega+\frac{q^{2}}{2}\right)^{2}, \\
\frac{k_{F}^{2}}{2}-\frac{1}{2 q^{2}}\left(\omega-\frac{q^{2}}{2}\right)^{2}, & \text { if } \frac{k_{F}^{2}}{2}<\frac{1}{2 q^{2}}\left(\omega+\frac{q^{2}}{2}\right)^{2}, \\
0, & \text { if } \frac{k_{F}^{2}}{2}<\frac{1}{2 q^{2}}\left(\omega-\frac{q^{2}}{2}\right)^{2},
\end{array}\right.
$$

with $\theta$ as the Heaviside step function. Introducing the Bogoliubov dispersion (4) and the interaction amplitude (5) in the expression for the imaginary part of the density-response function (17) leads to

$$
\operatorname{Im} \chi(\omega, \vec{k})=\frac{\alpha}{16 \pi \omega^{4}}\left(\frac{m_{B}+1}{m_{B}}\right)^{2} \sum_{\vec{q}}(\vec{k} \cdot \vec{q})^{2} \frac{q^{3}}{\sqrt{q^{2}+2}} S\left(\vec{k}+\vec{q}, \omega-\omega_{\vec{q}}\right) .
$$

In Fig. 2, expression (24) is shown as a function of $\omega$ for various momentum exchanges. A peak is seen that represents the emission of Bogoliubov excitations and which is shifted to higher frequencies for larger momentum exchanges. This behavior is to be expected since more energy is needed to create a Bogoliubov excitation with a higher momentum.

In Fig. 3(a), we have zoomed in on the peak for small values of momentum exchange, which reveals a shoulder structure on the main peak. This behavior also was observed in the context of the optical absorption of Fröhlich solid-state polarons in Refs. [27] and [17]. It was a consequence of the Pauli exclusion principle, which provided a constraint on the number of fermions that can participate in the Bragg scattering process, and in Ref. [27], it was shown that a maximal allowed participation was reached for $\omega=\omega_{\vec{k}}+\omega_{F}$ [with $\omega_{F}=\hbar k_{F} /\left(2 m_{I}\right)$, the Fermi frequency]. This phenomenon

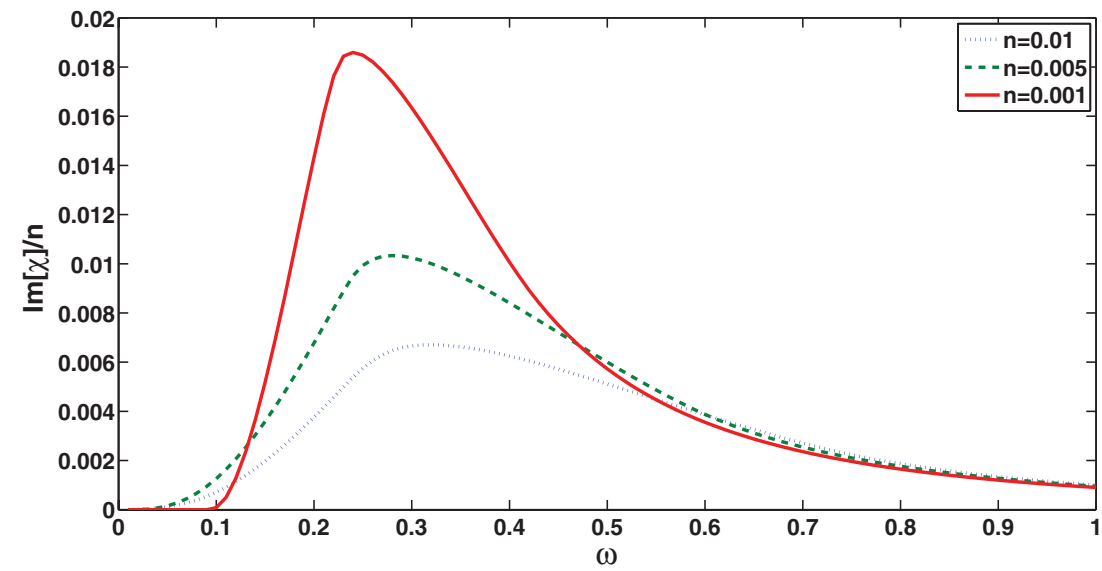

$063612-5$
FIG. 4. (Color online) The Bragg response (24) of the polaronic system consisting of polarized lithium- 6 impurities in a sodium condensate as a function of $\omega$ for different impurity densities $n$. The exchanged momentum is taken as $k=1$, and the polaronic-coupling parameter is $\alpha=$ 0.01 . Everything is in polaronic units, i.e., $\hbar=$ $m_{I}=\xi=1$. 


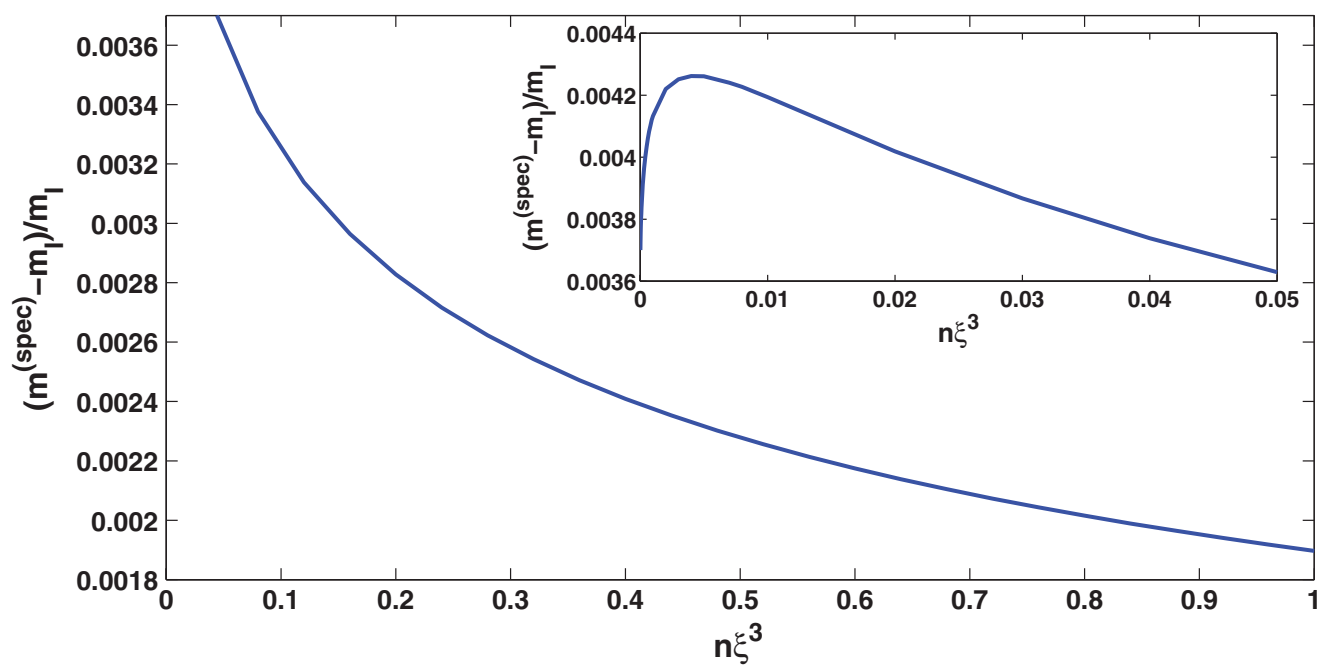

FIG. 5. (Color online) The spectral effective mass of the polarons as a function of the impurity density for lithium-6 impurities in a sodium condensate at $\alpha=0.01$ as determined by the sum rule (19).

provides a redistribution of the weight of the main peak toward this value, which explains the shoulder structure in Fig. 3(a). At larger values of the exchanged momentum $k$, the effect diminishes where we, thus, expect the peak to shift according to the Bogoliubov dispersion (4). In Fig. 3(b), the location of the maximum of the peak is plotted as a function of $k$ together with the Bogoliubov dispersion (4), which indeed is a better fit at larger $k$. At smaller $k$, we observe a deviation toward the Fermi frequency, as expected from this Pauli blocking mechanism.

In Fig. 4, the peak in the Bragg response is presented for different impurity densities. Note that, for higher densities, the spectral weight of the $\omega>0$ peak diminishes. This is not in contrast with the $f$-sum rule: The spectral weight of the $\omega=0 \delta$ peak in the spectrum compensates, in accordance with expression (19). For $\vec{k} \rightarrow 0$, the weight of this $\delta$ function is related to the spectral effective mass such that an attenuation of the peak at $\omega>0$ corresponds to a decrease of the spectral effective mass. The spectral effective mass deduced with the sum rule (19) is presented in Fig. 5 as a function of the impurity density. Note that the spectral effective mass generally behaves only qualitatively the same as the inertial effective mass from Fig. 1, and only in the limit $n \rightarrow 0$ is the same result retrieved. This is because the spectral effective mass is a one-particle property while the inertial effective mass is related to the entire polaronic system.

We would like to emphasize that, according to our calculations, different definitions of the effective mass result in another behavior and, in general, cannot be compared. This also means that, for an experiment, it is important to know which effective mass is of importance for the specific setup.

\section{CONCLUSIONS}

The weak-coupling many-polaron formalism, which was developed in the context of Fröhlich solid-state polarons in Refs. [15] and [17], was applied in the present paper to the case of the polaronic system consisting of impurities in a BEC. The properties of the ground state and the response to Bragg spectroscopy were examined. The sum rule of Ref. [24], which related the response to the spectral effective mass was formulated in the present context. Also, a calculation of the inertial effective mass was presented, which is related to the many-polaron system as a whole. It turns out that the ground-state properties are determined by the static structure factor of the impurities, while the response is governed by the dynamic structure factor as in the case of the Fröhlich solid-state polarons.

This generalization of the many-polaron formalism then is applied to the case of spin-polarized fermionic impurities, which behaves as an ideal gas for which the structure factors are well known. The numerical calculations were performed for lithium-6 impurities in a sodium condensate. Both the groundstate properties and the Bragg response indicate that, in the limit of high-impurity density, the polaron effect disappears, which is also the case for Fröhlich solid-state polarons. In the Bragg response, a peak is observed that corresponds to the emission of Bogoliubov excitations. The behavior of this peak, as a function of the exchanged momentum and the impurity density, was examined. Also, it is shown that the two definitions for the effective mass exhibit a different behavior; this is because the inertial effective mass is a property of the system as a whole while the spectral effective mass is a one-particle property.
[1] I. Bloch, J. Dalibard, and W. Zwerger, Rev. Mod. Phys. 80, 885 (2008).

[2] F. M. Cucchietti and E. Timmermans, Phys. Rev. Lett. 96, 210401 (2006).
[3] K. Sacha and E. Timmermans, Phys. Rev. A 73, 063604 (2006).

[4] L. D. Landau and S. I. Pekar, Zh. Eksp. Teor. Fiz. 18, 419 (1948).

[5] H. Fröhlich, Adv. Phys. 3, 325 (1954). 
[6] A. S. Alexandrov and J. T. Devreese, Advances in Polaron Physics (Springer, Heidelberg, 2009).

[7] M. Bruderer, A. Klein, S. R. Clark, and D. Jaksch, Phys. Rev. A 76, 011605 (2007).

[8] M. Bruderer, A. Klein, S. R. Clark, and D. Jaksch, New J. Phys. 10, 033015 (2008).

[9] A. Privitera and W. Hofstetter, Phys. Rev. A 82, 063614 (2010).

[10] J. Tempere, W. Casteels, M. K. Oberthaler, S. Knoop, E. Timmermans, and J. T. Devreese, Phys. Rev. B 80, 184504 (2009).

[11] W. Casteels, J. Tempere, and J. T. Devreese, Phys. Rev. A 83, 033631 (2011).

[12] W. Casteels, T. Van Cauteren, J. Tempere, and J. Devreese, Laser Phys. 21, 1480 (2011).

[13] A. Sumi and Y. Toyozawa, J. Phys. Soc. Jpn. 35, 137 (1973).

[14] T. D. Lee, F. E. Low, and D. Pines, Phys. Rev. 90, 297 (1953).

[15] L. F. Lemmens, F. Brosens, and J. H. Devreese, Phys. Status Solidi B 82, 439 (1977).

[16] J. Devreese, J. De Sitter, and M. Goovaerts, Phys. Rev. B 5, 2367 (1972).

[17] J. Tempere and J. T. Devreese, Phys. Rev. B 64, 104504 (2001).
[18] L. Pitaevskii and S. Stringari, Bose-Einstein Condensation, 1st ed. (Oxford University Press, Oxford, 2003).

[19] L. Viverit, C. J. Pethick, and H. Smith, Phys. Rev. A 61, 053605 (2000).

[20] M. J. Bijlsma, B. A. Heringa, and H. T. C. Stoof, Phys. Rev. A 61, 053601 (2000).

[21] D. M. Stamper-Kurn, A. P. Chikkatur, A. Görlitz, S. Inouye, S. Gupta, D. E. Pritchard, and W. Ketterle, Phys. Rev. Lett. 83, 2876 (1999).

[22] J. Steinhauer, R. Ozeri, N. Katz, and N. Davidson, Phys. Rev. Lett. 88, 120407 (2002).

[23] S. Nascimbène, N. Navon, K. J. Jiang, L. Tarruell, M. Teichmann, J. McKeever, F. Chevy, and C. Salomon, Phys. Rev. Lett. 103, 170402 (2009).

[24] J. T. Devreese, L. F. Lemmens, and J. Van Royen, Phys. Rev. B 15, 1212 (1977).

[25] J. L. M. van Mechelen, D. van der Marel, C. Grimaldi, A. B. Kuzmenko, N. P. Armitage, N. Reyren, H. Hagemann, and I. I. Mazin, Phys. Rev. Lett. 100, 226403 (2008).

[26] D. Pines, Many Body Problem (Benjamin, New York, 1962).

[27] X. Wu, F. M. Peeters, and J. T. Devreese, Phys. Rev. B 34, 2621 (1986).

[28] J. Tempere and J. Devreese, Eur. Phys. J. B 20, 27 (2001).

[29] G. D. Mahan, Many-Particle Physics, 2nd. ed (Plenum, New York, 1990). 